\title{
Article \\ Comparison of Organic and Inorganic Mulching for Weed Suppression in Wheat under Rain-Fed Conditions of Haripur, Pakistan
}

\author{
Sami Ullah Khan ${ }^{1}$, Xiukang Wang ${ }^{2, *(\mathbb{C})}$, Tariq Mehmood ${ }^{1}$, Sohail Latıf ${ }^{3}$, Saftain Ullah Khan ${ }^{4}$, Sajid Fiaz ${ }^{5}$ (D) \\ and Abdul Qayyum 1,*(i) \\ 1 Department of Agronomy, The University of Haripur, Haripur 22620, Pakistan; \\ samiullah@uoh.edu.pk (S.U.K.); mehmood143@gmail.com (T.M.) \\ 2 College of Life Sciences, Yan'an University, Yan'an 716000, China \\ 3 Research Farm Crops, Baldmas District Kotli, Agriculture Department (Research Wing), Government of Azad \\ Jammu and Kashmir, Kotli 11100, Pakistan; sohaillatif1975@gmail.com \\ 4 Soil and Water Testing Laboratory, Mianwali 42200, Pakistan; saftainkhan6042@gmail.com \\ 5 Department of Plant Breeding and Genetics, The University of Haripur, Haripur 22620, Pakistan; \\ sfiaz@uoh.edu.pk \\ * Correspondence: wangxiukang@yau.edu.cn (X.W.); aqayyum@uoh.edu.pk (A.Q.)
}

check for

updates

Citation: Khan, S.U.; Wang, X.; Mehmood, T.; Latıf, S.; Khan, S.U.; Fiaz, S.; Qayyum, A. Comparison of Organic and Inorganic Mulching for Weed Suppression in Wheat under Rain-Fed Conditions of Haripur, Pakistan. Agronomy 2021, 11, 1131. https://doi.org/10.3390/

agronomy11061131

Academic Editor: Stéphane Cordeau

Received: 26 April 2021

Accepted: 31 May 2021

Published: 2 June 2021

Publisher's Note: MDPI stays neutral with regard to jurisdictional claims in published maps and institutional affiliations.

Copyright: (c) 2021 by the authors. Licensee MDPI, Basel, Switzerland. This article is an open access article distributed under the terms and conditions of the Creative Commons Attribution (CC BY) license (https:/ / creativecommons.org/licenses/by/ $4.0 /)$.

\begin{abstract}
Weeds pose a great threat to crop production subsequently distressing the equilibrium of agro-ecological systems globally. Instead of relying on chemical weed control, there is a dire need to explore alternative eco-friendly agricultural practices for weed suppression and sustainable wheat production. Mulching being eco-friendly could potentially serve the purpose towards weed suppression organically. To check the usefulness of mulching, two-year studies were conducted under field conditions in Haripur, Pakistan, during 2014 and 2015 to elucidate the response of various mulching materials for weed control in wheat. Six mulch material treatments were used along with Buctril super (used as check) at the rate of $1.235 \mathrm{~L} \mathrm{ha}^{-1}$ to control the most problematic weed species of wheat in Pakistan. Experiments were designed under randomized complete block design with four repeats. The analysis of data revealed a significant decrease in weed density, relative weed density, fresh and dry plant biomass at 25,50 and 75 days after sowing (DAS) where Buctril super at 1.235 $\mathrm{L} \mathrm{ha}^{-1}$ and mulch of black plastic were used followed by sugarcane bagasse and dry leaves of mulberry, as compared with control (untreated). Higher density of weed, relative weed density, fresh and dry plant biomass were observed in test weed species of wheat where lentil was applied with wheat and dry leaves of mulberry were incorporated. Net economic benefits in the form of benefit cost ratio (2.55) were higher where grass clippings were applied followed by sugarcane bagasse (2.43), mulberry leaves (2.49), while the lowest net economic benefits (1.72) were obtained when lentil (grown as live mulch crop) with wheat was intercropped. It was concluded from the study that sugarcane bagasse and grass clippings could be a source to control weeds in the wheat with a minimum cost of production at Haripur, Pakistan, and similar agro-climatic environment.
\end{abstract}

Keywords: wheat; weed suppression; inorganic mulch; economic benefits; organic mulch

\section{Introduction}

Wheat (Triticum aestivum L.) is considered as a staple food for almost $33 \%$ of the world's population [1]. Wheat is produced in irrigated and unirrigated areas in Pakistan. Its cultivation area in rain-fed areas of Pakistan is 1.24 million hectares with average yield of $0.5-1.3 \mathrm{t} \mathrm{ha}^{-1}$ but in irrigated areas, average yield is about $2.7 \mathrm{t} \mathrm{ha}^{-1}$ in spite of genetic yield potential of $6-8 \mathrm{tha}^{-1}$. There are several factors which reduce wheat yield including weed invasion as significant problem in yield reduction up to $40 \%$ [2]. Prominent weed species troublesome for wheat production in Pakistan are Avena fatua, Carthamus oxyacantha, Chenopodium album, Convolvulus arvensis, Euphorbia helioscopia, Fumaria indica, Phalaris minor 
and Sonchus oleraceus. The present agricultural system in the country is dependent on inorganic/synthetic herbicides to manage or eradicate crop weeds [3]. Use of synthetic herbicides causes health and ecologically associated problems. That is why it is very important to develop biological methods for weed management. In this regard, allelopathy is a novel option in modern agriculture that can be applied in a weed control management system [4].

Previous reports showed weed management in different crops through allelopathy such as canola (Brassica napus L.), cotton (Gossypium hirsutum L.), rice (Oryza sativa L.), wheat and maize $[5,6]$. Several potential allelopathic crops such as sunflower (Helianthus annus L.), rice (Oryza sativa L.), sorghum (Sorghum bicolor L.), brassica (Brassica campestris L.), tobacco (Nicotiana tabacum L.), sesame (Sesamum indicum L.) and trees such as eucalyptus (Eucalyptus camaldulensis D.) and mulberry (Morus spp.) are known for having allelopathic effects $[7,8]$. These plants contain allelochemicals such as benzoic acid, syringic acid, ferulic acid, gallic acid, protocateuic acid, $m$-coumaric acid, caffeic acid, vanillic acid, $p$ hydroxybenzoic acid, $p$-coumaric acid, dhurrin, $p$-sorgoleone and hydroxybenzaldehyde whose extracts have been applied to suppress target weeds $[9,10]$.

Allelopathic weed control can be realized by planting allelopathic plants in the close vicinity of weeds which stimulate production of these chemicals (Tesio and Ferrero, 2010). The decomposing plant material releases allelochemicals which are absorbed by the target weeds. The most important example for such cases includes the use of allelopathic plant residues for weed control. Allelopathic weed control can also be applied by growing allelopathic plants in a field for a certain period of time, in order for their roots to exude allelochemicals [11]. Cover crops with allelopathic ability can destroy weeds. Some important cover crops include cereal rye, canola, wheat, rape seed, crimson clover, brown mustard, red clover, cowpea, oats, fodder radish, buckwheat, hairy vetch, and black mustard. Some of the cropping systems such as organic cropping depend on cover cropping for weed suppression [12].

Mulches used as cover crops and intercropping (as live crop grown in between the rows of main crop) provide different benefits in agro-ecologies through competition and allelopathy such as nitrogen fixation, erosion control, improving organic matter, nutrient recycling, pest and weed control, and improving soil organism [13]. Water extracts of plants having allelopathic effects or mulches of sorghum, sunflower, rice, sesame, tobacco, eucalyptus and mulberry suppressed weed growth by $22-46 \%$ and boosted wheat yield by $17-21 \%$ [14]. Data about the allelopathic potential of maize stalk mulch, dry leaves of mulberry, sugarcane bagasse, grass clippings and lentil used as intercrop through competition used for organic weed control is limited in wheat crops under arid conditions. This experiment was conducted to investigate the effectiveness of various organic and inorganic mulches along with a bared ground control with pre-emergence herbicide on weed suppression in wheat under rain-fed conditions. The study was conducted also to switch over to organic agriculture and find farmer friendly economical practices to manage weeds in wheat.

\section{Materials and Methods}

\subsection{Experiment Details}

The study was conducted at farmer field village Mang $\left(31.25^{\circ} \mathrm{N}, 73.06^{\circ} \mathrm{E}\right.$ and $183 \mathrm{~m}$ asl), District Haripur Khyber Pakhtunkhwa, Pakistan, in November 2013-2014 and 20142015. The study was performed in a randomized complete block design (RCBD) with factorial arrangement having four repeats. Plot $(5 \mathrm{~m} \times 3 \mathrm{~m})$ had row spacing of $30 \mathrm{~cm}$. Before sowing of crop, soil samples $(0-30 \mathrm{~cm}$ depth) were collected from various locations of the experimental field. Composite soil samples were air dried, ground and passed through $2 \mathrm{~mm}$. The physico-chemical characteristics of the soil were estimated by the hydrometer method [15]. Phosphorus and potassium (AB-DTPA extractable) were estimated by the procedure described by Soltanpour and Schwab [16] using spectrophotometer and flame photometer. The Kjeldhal method of Bremner [17] was used for calculation of total 
nitrogen in the soil. Soil organic matter was estimated by the procedure as described by BALL [18]. The DTPA (diethylenetriaminepentaacetic acid) micronutrient extraction procedure of Lindsay and Norvell [19] was used for estimation of soil zinc, copper, iron and manganese. Analysis of soil for physiochemical properties revealed texture silt loam alkaline with $\mathrm{pH} 7.1$, electrical conductivity $0.29 \mathrm{dSm}^{-1}$, organic matter $1.02 \%$, nitrogen $0.051 \%$,

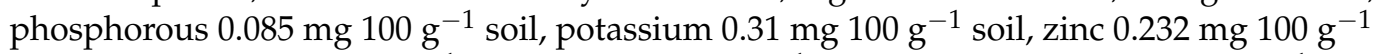

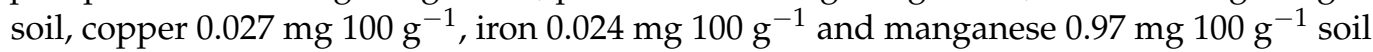
respectively. The weather data of the experimental site (2013-2014 and 2014-2015) is shown in Figures 1 and 2, respectively.

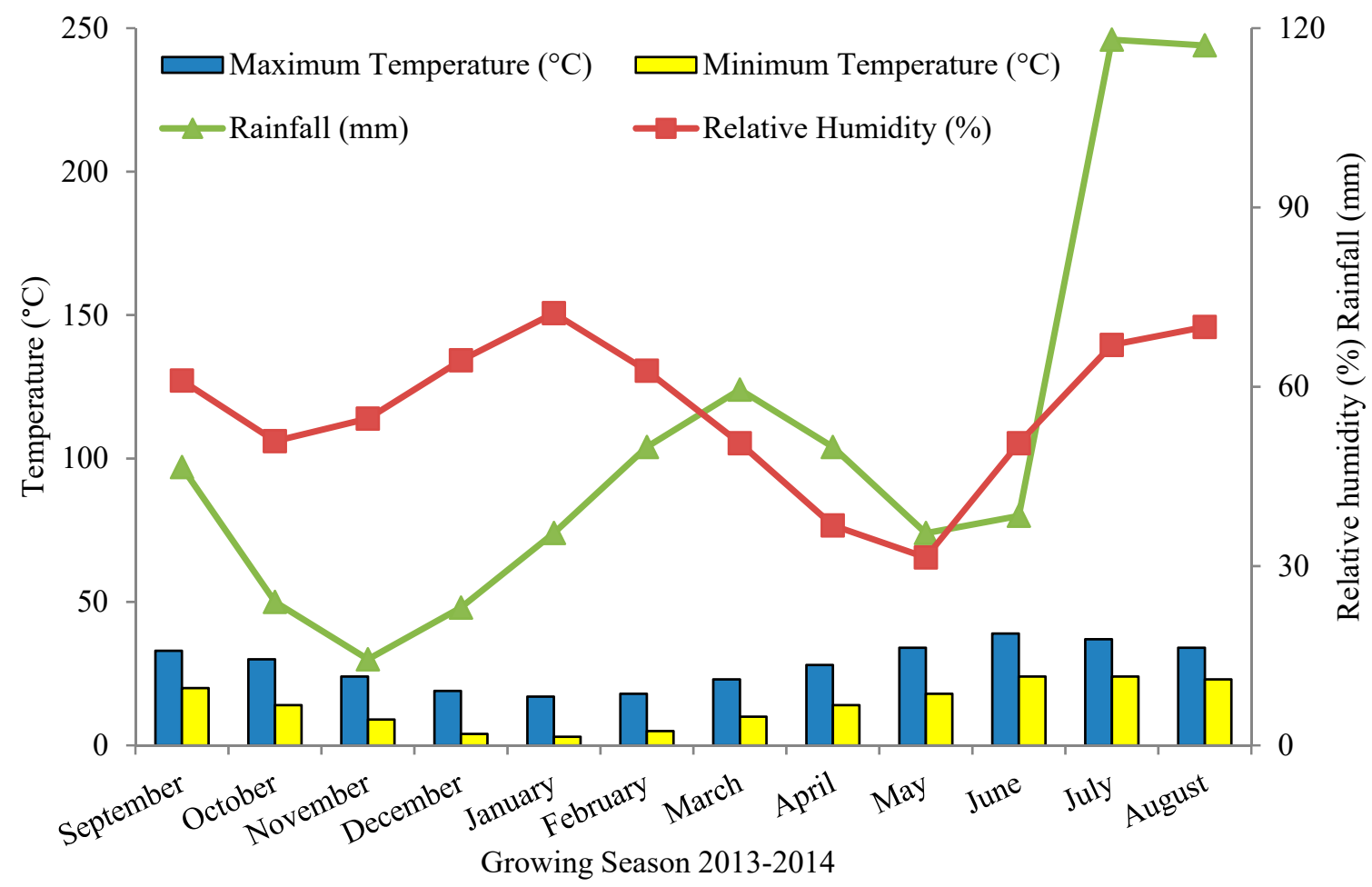

Figure 1. Meteorological data regarding average minimum and maximum temperatures $\left({ }^{\circ} \mathrm{C}\right)$, monthly rainfall (mm) and relative humidity (\%) of 2013-2014.

Seeds of wheat cultivar Pirsabak-2005 were obtained from the Cereal Crop Research Institute, Pirsabak, Nowshera, and sown in lines by a hand drill using seed rate of $125 \mathrm{~kg} \mathrm{ha}^{-1}$ during first week of November, 2013 and 2014. Treatments were designed as T1: Control (No mulching), T2: Maize stalk mulch @ $4 \mathrm{tha}^{-1}$, T3: Dry leaves of mulberry @ $4 \mathrm{t} \mathrm{ha}^{-1}$, T4: Sugarcane bagasse @ $4 \mathrm{t} \mathrm{ha}^{-1}$, T5: Grass clippings @ $4 \mathrm{tha}^{-1}$, T6: Living mulch (lentil crop), T7: Mulch of black plastic and T8: Herbicide (Buctril Super @ 1.235 L ha ${ }^{-1}$ ). Maize stalk, dry leaves of mulberry, sugarcane bagasse, mulch of black plastic and grass clippings were applied after sowing of crops while living mulch (lentil crop) was applied in between rows of the main crop (wheat) after sowing while herbicide was applied after sowing of wheat. 


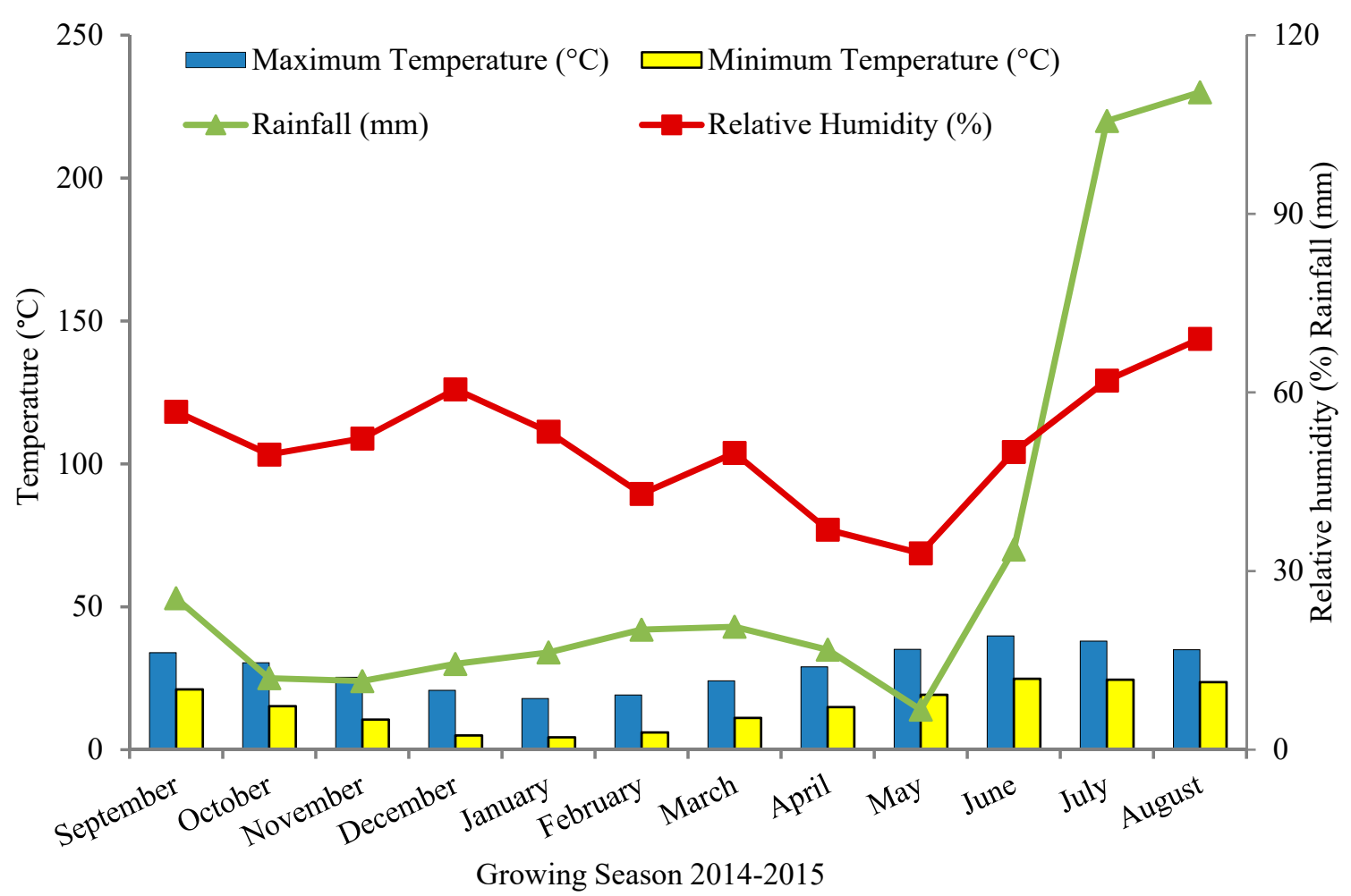

Figure 2. Meteorological data regarding average minimum and maximum temperatures $\left({ }^{\circ} \mathrm{C}\right)$, monthly rainfall (mm) and relative humidity (\%) of 2014-2015.

Phosphorus (100 kg ha $\left.{ }^{-1}\right)$ form di-ammonium phosphate, potash $\left(100 \mathrm{~kg} \mathrm{ha}^{-1}\right)$ form sulfate of potash and nitrogen $\left(150 \mathrm{~kg} \mathrm{ha}^{-1}\right)$ form ammonium sulphate was applied in two splits: half at the time of sowing while the remaining nitrogen was applied at tillering stage. Crop was harvested manually on 20 April 2014 and 2015. Leaf area of wheat plants were measured using portable leaf area meter (CI-202, CID Bio-Science Inc., Portland, OR, USA). Plants were sun dried after harvesting, weighed and biological yield was recorded using spring balance. The plots were separately threshed and grain yield was determined by electrical balance.

\subsection{Sampling and Observations}

Weeds density $\left(\mathrm{m}^{-2}\right)$ at 25,50 and 75 days after sowing (DAS) was noted through quadrates of $50 \times 50 \mathrm{~cm}\left(0.25 \mathrm{~m}^{-2}\right)$ from every plot at 25, 50 and 75 DAS $(\mathrm{BBCH})$ at principal growth stages 2,4 and 7 [20]. The weeds species found in the quadrat were identified and their number was noted by uprooting from the ground. The numbers of weeds were counted by randomly throwing quadrat at two different locations in each plot and then the average was taken.

The relative weed density $\left(\mathrm{m}^{2}\right)$ at 25,50 and 75 DAS was determined using the following formula:

$$
\text { Relative weed density }(\mathrm{RD})=\frac{\text { Density of a given weed species }}{\text { Density of total weeds }} \times 100
$$

Weed dry biomass was noted in quadrats of $50 \times 50 \mathrm{~cm}\left(0.25 \mathrm{~m}^{-2}\right)$ at 25,50 and 75 DAS. Weeds were clipped off, put in paper bags and oven dried at $70^{\circ} \mathrm{C}$ for $72 \mathrm{~h}$. After drying, weed dry weight was measured using a digital balance $(T \times 323 \mathrm{~L}$, Shimadzu, Japan). 


\subsection{Economic Analysis}

Economic analysis of different treatments, cost of wheat production (seedbed preparation, tillage, sowing, land rent, watch and ward, cost of fertilizers, harvesting and threshing was calculated etc.) in the years 2014 and 2015 was calculated [21]. The variable cost incurring from various mulching materials and herbicide use during the two growing seasons was calculated. The gross income was determined on the basis of wheat yield per hectare according to prevailing market value (Source: Agriculture Policy Institute, Islamabad, Pakistan). Benefits cost ratio (BCR) was calculated for all individual treatments using the following formula and best treatment contributing towards wheat income was identified.

$$
\mathrm{BCR}=\frac{\text { Gross income }}{\text { Total cost }}
$$

After BCR calculation, net income (USD ha ${ }^{-1}$ ) was worked out for each treatment by the following formula:

$$
\text { Net income }\left(\mathrm{USD} \mathrm{ha}^{-1}\right)=\text { Gross income }- \text { Total variable cost }
$$

\subsection{Statistical Analysis}

The collected data were analysed by analysis of variance (ANOVA) and means were compared by employing Tukey's honestly significant difference (HSD) test at $5 \%$ probability using Statistix 8.1.

\section{Results}

\subsection{Mulching Materials Effect on Weed Density}

Higher reduction (up to $96 \%$ ) in weed density of all the tested weeds was observed under Herbicide spray at 25, 50 and 75 DAS respectively. Maximum weed density reduction (93\%) of Convolvulus arvensis on 25 DAS was observed where mulch of black plastic and herbicide spray were applied separately (Table 1). Dry leaves of mulberry and living mulch affect were minimum on weed density of all weeds at all the three stages of application (Table 1). The overall trend of weed density reduction by various mulching treatments was in the following order: herbicide spray $>$ black plastic mulch $>$ sugarcane bagasse $>$ maize stalk mulch $>$ grass clipping $>$ living mulch $>$ dry leaves of mulberry. The magnitude of reduction (up to $90 \%$ ) in weed density by black plastic mulch was noted comparable to herbicide spray at all the three growth stages.

\subsection{Mulching Materials Effect on Relative Weed Density}

Lowest relative weed density of Carthamus oxyacantha and Convolvulus arvensis was observed where herbicide was sprayed and it was statistically similar to mulch of black plastic, sugarcane bagasse and maize stalk (Table 2). Maximum relative weed density was also noticed in dry leaves of mulberry treatment and found statistically $(p \leq 0.05)$ similar to mulching of lentil intercropped with wheat and control. The overall maize stalk mulch was found best to reduce the relative weed densities of the tested weeds at 25, 50 and 75 DAS. However, the effect of grass clippings was highest in reducing relative weed densities of Euphorbia helioscopia, Fumaria indica, Phalaris minor and Sonchus oleraceus at all the three growth stages (Table 2). 
Table 1. Effect of various mulch materials applied on weed density $\left(\right.$ weeds $\mathrm{m}^{-2}$ ) of different weed species of wheat.

\begin{tabular}{|c|c|c|c|c|c|c|c|c|c|c|c|c|}
\hline \multirow{2}{*}{ Treatments } & \multicolumn{3}{|c|}{ Avena fatua } & \multicolumn{3}{|c|}{ Carthamus oxyacantha } & \multicolumn{3}{|c|}{ Chenopodium album } & \multicolumn{3}{|c|}{ Convolvulus arvensis } \\
\hline & 25 DAS & 50 DAS & 75 DAS & 25 DAS & 50 DAS & 75 DAS & 25 DAS & 50 DAS & 75 DAS & 25 DAS & 50 DAS & 75 DAS \\
\hline Control & $9.75 \pm 0.97 \mathrm{a}$ & $10.07 \pm 0.90 \mathrm{a}$ & $11.90 \pm 0.88 \mathrm{a}$ & $7.60 \pm 0.96 \mathrm{a}$ & $8.00 \pm 1.20 \mathrm{a}$ & $20.00 \pm 1.50 \mathrm{a}$ & $11.87 \pm 1.87 \mathrm{a}$ & $10.85 \pm 1.77 a$ & $9.05 \pm 0.99 \mathrm{a}$ & $18.25 \pm 1.89 \mathrm{a}$ & $10.15 \pm 1.25 \mathrm{a}$ & $14.42 \pm 1.27 \mathrm{a}$ \\
\hline Maize stalk mulch & $3.05 \pm 0.41 \mathrm{c}$ & $3.00 \pm 0.51 \mathrm{c}$ & $3.70 \pm 0.21 \mathrm{c}$ & $3.00 \pm 0.55 c$ & $2.12 \pm 0.41 \mathrm{c}$ & $5.35 \pm 0.77 \mathrm{bc}$ & $12.93 \pm 2.09 \mathrm{a}$ & $3.50 \pm 1.05 c$ & $3.07 \pm 0.78 \mathrm{c}$ & $14.77 \pm 1.08 \mathrm{bc}$ & $3.12 \pm 0.78 \mathrm{c}$ & $5.47 \pm 0.87 \mathrm{bc}$ \\
\hline Dry leaves of mulberry & $8.12 \pm 0.82 \mathrm{a}$ & $9.77 \pm 0.68 \mathrm{a}$ & $10.70 \pm 0.2 \mathrm{a}$ & $6.00 \pm 0.77 \mathrm{ab}$ & $6.82 \pm 1.07 \mathrm{a}$ & $18.27 \pm 1.20 \mathrm{a}$ & $12.93 \pm 1.51 \mathrm{a}$ & $7.70 \pm 1.55 \mathrm{~b}$ & $8.42 \pm 1.55 \mathrm{a}$ & $14.75 \pm 1.05 \mathrm{bc}$ & $9.87 \pm 1.29 \mathrm{a}$ & $13.55 \pm 1.21 \mathrm{a}$ \\
\hline Sugarcane bagasse & $1.75 \pm 0.12 \mathrm{~cd}$ & $1.75 \pm 0.31 \mathrm{c}$ & $1.75 \pm 0.11 \mathrm{~d}$ & $1.10 \pm 0.33 \mathrm{~d}$ & $0.25 \pm 0.05 \mathrm{~d}$ & $3.07 \pm 0.66 \mathrm{~cd}$ & $12.05 \pm 2.01 \mathrm{a}$ & $1.62 \pm 1.01 \mathrm{cde}$ & $1.25 \pm 0.65 \mathrm{~cd}$ & $1.85 \pm 0.45 \mathrm{bc}$ & $1.25 \pm 0.45 \mathrm{~d}$ & $1.87 \pm 0.17 \mathrm{~cd}$ \\
\hline Grass clippings & $6.12 \pm 0.77 b$ & $5.65 \pm 0.70 \mathrm{~b}$ & $5.02 \pm 0.49 c$ & $1.50 \pm 0.19 \mathrm{~cd}$ & $3.70 \pm 0.27 \mathrm{bc}$ & $7.60 \pm 0.60 \mathrm{~b}$ & $10.57 \pm 1.19 \mathrm{a}$ & $3.00 \pm 1.08 \mathrm{~cd}$ & $5.35 \pm 0.88 b$ & $10.57 \pm 1.01 \mathrm{~d}$ & $4.70 \pm 0.98 \mathrm{c}$ & $6.02 \pm 0.55 b$ \\
\hline Living mulch (lentil crop) & $9.10 \pm 1.10 \mathrm{a}$ & $10.07 \pm 1.03 a$ & $8.00 \pm 1.00 \mathrm{~b}$ & $6.82 \pm 0.58 \mathrm{a}$ & $5.00 \pm 0.87 b$ & $17.67 \pm 1.05 \mathrm{a}$ & $10.45 \pm 1.96 \mathrm{a}$ & $6.75 \pm 1.55 b$ & $7.22 \pm 1.23 \mathrm{a}$ & $16.05 \pm 1.87 \mathrm{~b}$ & $7.70 \pm 1.55 b$ & $13.55 \pm 1.01 \mathrm{a}$ \\
\hline Black plastic mulch & $1.50 \pm 0.21 \mathrm{~d}$ & $1.50 \pm 0.09 c$ & $0.75 \pm 0.07 \mathrm{~d}$ & $0.75 \pm 0.63 \mathrm{~d}$ & $0.25 \pm 0.41 \mathrm{~d}$ & $1.52 \pm 0.40 \mathrm{~d}$ & $9.60 \pm 1.22 \mathrm{a}$ & $1.00 \pm 0.78 \mathrm{de}$ & $1.00 \pm 0.65 \mathrm{~d}$ & $1.27 \pm 0.23 \mathrm{e}$ & $1.12 \pm 0.29 \mathrm{~d}$ & $2.05 \pm 0.55 \mathrm{~cd}$ \\
\hline Herbicide spray & $1.07 \pm 0.18 \mathrm{~d}$ & $1.25 \pm 0.16 \mathrm{c}$ & $0.55 \pm 0.07 \mathrm{~d}$ & $0.75 \pm 0.07 \mathrm{~d}$ & $0.18 \pm 0.03 \mathrm{~d}$ & $1.12 \pm 0.34 \mathrm{~d}$ & $13.97 \pm 1.75 \mathrm{a}$ & $0.25 \pm 0.11 \mathrm{de}$ & $0.75 \pm 0.11 \mathrm{~d}$ & $1.25 \pm 0.21 \mathrm{e}$ & $0.50 \pm 0.07 \mathrm{~d}$ & $1.12 \pm 0.23 \mathrm{~d}$ \\
\hline LSD at $0.05 P$ & 1.48 & 1.78 & 1.89 & 1.68 & 1.63 & 2.35 & NS & 2.11 & 1.85 & 1.34 & 1.71 & 3.60 \\
\hline Control & $9.10 \pm 1.22 \mathrm{a}$ & $9.75 \pm 1.46 \mathrm{a}$ & $10.67 \pm 0.99 \mathrm{a}$ & $7.70 \pm 0.49 \mathrm{a}$ & $6.17 \pm 1.06 \mathrm{a}$ & $10.17 \pm 1.55 \mathrm{a}$ & $8.62 \pm 0.93 \mathrm{a}$ & $9.42 \pm 1.56 \mathrm{a}$ & $11.25 \pm 1.39 \mathrm{a}$ & $8.72 \pm 0.89 a$ & $11.27 \pm 1.88 \mathrm{a}$ & $16.25 \pm 1.45 \mathrm{a}$ \\
\hline Maize stalk mulch & $2.25 \pm 0.89 c$ & $3.37 \pm 0.56 c$ & $4.05 \pm 0.63 c$ & $2.62 \pm 0.72 c$ & $3.37 \pm 0.78 \mathrm{bc}$ & $2.77 \pm 0.41 \mathrm{~d}$ & $3.70 \pm 0.66 \mathrm{~cd}$ & $3.25 \pm 0.73 b$ & $4.60 \pm 0.67 c$ & $1.25 \pm 0.21 \mathrm{~b}$ & $8.40 \pm 1.12 \mathrm{a}$ & $9.37 \pm 1.07 \mathrm{bc}$ \\
\hline Dry leaves of mulberry & $8.20 \pm 1.09 \mathrm{ab}$ & $9.12 \pm 1.98 \mathrm{a}$ & $9.45 \pm 1.45 \mathrm{a}$ & $6.37 \pm 1.04 \mathrm{ab}$ & $6.02 \pm 0.99 a$ & $9.60 \pm 1.09 \mathrm{ab}$ & $6.00 \pm 0.76 \mathrm{~b}$ & $8.60 \pm 1.91 \mathrm{a}$ & $11.05 \pm 1.87 \mathrm{a}$ & $8.32 \pm 0.56 \mathrm{a}$ & $10.75 \pm 1.01 \mathrm{a}$ & $14.62 \pm 1.17 \mathrm{a}$ \\
\hline Sugarcane bagasse & $1.75 \pm 0.41 \mathrm{c}$ & $1.50 \pm 0.55 \mathrm{~d}$ & $1.50 \pm 0.41 \mathrm{~d}$ & $1.82 \pm 0.42 \mathrm{~cd}$ & $1.00 \pm 0.45 \mathrm{~d}$ & $1.50 \pm 0.29 \mathrm{de}$ & $2.00 \pm 0.42 \mathrm{de}$ & $1.65 \pm 0.85 c$ & $0.19 \pm 0.03 \mathrm{~d}$ & $1.50 \pm 0.09 \mathrm{~b}$ & $9.32 \pm 1.19 \mathrm{a}$ & $5.00 \pm 0.77 \mathrm{bc}$ \\
\hline Grass clippings & $6.70 \pm 0.87 b$ & $5.12 \pm 0.97 b$ & $6.35 \pm 0.88 \mathrm{~b}$ & $2.75 \pm 0.56 c$ & $4.40 \pm 0.78 \mathrm{ab}$ & $5.77 \pm 0.73 c$ & $5.10 \pm 1.25 b c$ & $3.57 \pm 0.77 b$ & $7.00 \pm 0.81 \mathrm{~b}$ & $1.50 \pm 0.14 \mathrm{~b}$ & $9.25 \pm 1.07 \mathrm{a}$ & $10.00 \pm 0.91 b c$ \\
\hline Living mulch (lentil crop) & $9.10 \pm 1.25 \mathrm{a}$ & $9.55 \pm 1.49 \mathrm{a}$ & $7.50 \pm 1.96 \mathrm{~b}$ & $7.30 \pm 1.23 \mathrm{a}$ & $6.17 \pm 0.71 \mathrm{a}$ & $8.50 \pm 1.21 \mathrm{~b}$ & $5.50 \pm 1.89 \mathrm{~b}$ & $9.42 \pm 1.54 \mathrm{a}$ & $10.50 \pm 0.88 \mathrm{a}$ & $7.37 \pm 0.78 \mathrm{a}$ & $11.27 \pm 1.45 \mathrm{a}$ & $12.92 \pm \mathrm{a} 0.88 \mathrm{~b}$ \\
\hline Black plastic mulch & $1.75 \pm 0.16 c$ & $1.00 \pm 0.38 \mathrm{~d}$ & $1.00 \pm 0.40 \mathrm{~d}$ & $1.25 \pm 0.71 \mathrm{~cd}$ & $1.50 \pm 0.14 \mathrm{~cd}$ & $1.05 \pm 0.63 \mathrm{e}$ & $2.00 \pm 0.63$ de & $1.25 \pm 0.65 c$ & $0.25 \pm 0.03 \mathrm{~d}$ & $1.00 \pm 0.56 \mathrm{~b}$ & $8.52 \pm 1.06 \mathrm{a}$ & $3.75 \pm 0.19 c$ \\
\hline Herbicide spray & $1.25 \pm 0.21 \mathrm{c}$ & $0.75 \pm 0.26 \mathrm{~d}$ & $0.75 \pm 0.09 \mathrm{~d}$ & $0.25 \pm 0.03 \mathrm{~d}$ & $1.25 \pm 0.19 \mathrm{~d}$ & $0.65 \pm 0.09 \mathrm{e}$ & $1.75 \pm 0.87 \mathrm{e}$ & $1.25 \pm 0.76 \mathrm{c}$ & $0.40 \pm 0.02 \mathrm{~d}$ & $0.75 \pm 0.19 b$ & $6.00 \pm 0.78 \mathrm{a}$ & $3.75 \pm 0.47 \mathrm{c}$ \\
\hline LSD at $0.05 P$ & 1.61 & 1.29 & 1.51 & 1.66 & 2.01 & 1.44 & 1.78 & 1.53 & 1.71 & 1.88 & NS & 7.96 \\
\hline
\end{tabular}

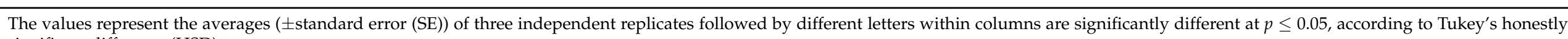
significant difference (HSD) test. 
Table 2. Effect of various mulch materials on relative weed density (\%) of different weed species of wheat.

\begin{tabular}{|c|c|c|c|c|c|c|c|c|c|c|c|c|}
\hline \multirow{2}{*}{ Treatments } & \multicolumn{3}{|c|}{ Avena fatua } & \multicolumn{3}{|c|}{ Carthamus oxyacantha } & \multicolumn{3}{|c|}{ Chenopodium album } & \multicolumn{3}{|c|}{ Convolvulus arvensis } \\
\hline & 25 DAS & 50 DAS & 75 DAS & 25 DAS & 50 DAS & 75 DAS & 25 DAS & 50 DAS & 75 DAS & 25 DAS & 50 DAS & 75 DAS \\
\hline Control & $23.42 \pm 1.78 \mathrm{a}$ & $15.82 \pm 0.98 \mathrm{ab}$ & $18.12 \pm 0.93 \mathrm{a}$ & $14.00 \pm 0.69 \mathrm{a}$ & $16.00 \pm 1.23 \mathrm{a}$ & $12.27 \pm 1.07 \mathrm{a}$ & $5.40 \pm 0.96 \mathrm{a}$ & $17.25 \pm 1.57 \mathrm{a}$ & $6.40 \pm 0.59 a$ & $8.47 \pm 1.03 \mathrm{a}$ & $13.87 \pm 1.11 \mathrm{a}$ & $9.97 \pm 1.13 \mathrm{a}$ \\
\hline $\begin{array}{l}\text { Maize stalk } \\
\text { mulch }\end{array}$ & $10.85 \pm 0.87 \mathrm{~b}$ & $10.85 \pm 0.55 \mathrm{~cd}$ & $9.22 \pm 0.87 \mathrm{~cd}$ & $5.87 \pm 0.81 \mathrm{ab}$ & $\begin{array}{l}7.52 \pm 0.91 \\
\text { abcd }\end{array}$ & $2.85 \pm 0.44 \mathrm{~d}$ & $1.30 \pm 0.18 \mathrm{~cd}$ & $12.93 \pm 1.08 \mathrm{ab}$ & $2.52 \pm 0.19 \mathrm{~d}$ & $2.25 \pm 0.51 \mathrm{~d}$ & $6.25 \pm 0.69 \mathrm{bc}$ & $4.50 \pm 0.88 \mathrm{~b}$ \\
\hline $\begin{array}{l}\text { Dry leaves of } \\
\text { mulberry }\end{array}$ & $18.52 \pm 1.58 \mathrm{ab}$ & $17.10 \pm 1.07 \mathrm{a}$ & $16.07 \pm 1.09 \mathrm{ab}$ & $7.57 \pm 0.94 \mathrm{ab}$ & $14.02 \pm 1.19 \mathrm{ab}$ & $11.17 \pm 0.85 \mathrm{a}$ & $2.62 \pm 0.28 \mathrm{bc}$ & $13.02 \pm 1.02 \mathrm{ab}$ & $5.80 \pm 0.53 \mathrm{ab}$ & $7.70 \pm 0.69 \mathrm{ab}$ & $9.30 \pm 0.51 \mathrm{ab}$ & $9.30 \pm 0.98 \mathrm{a}$ \\
\hline $\begin{array}{l}\text { Sugarcane } \\
\text { bagasse }\end{array}$ & $18.97 \pm 1.41 \mathrm{ab}$ & $9.00 \pm 0.59$ de & $6.10 \pm 0.76 \mathrm{~d}$ & $4.50 \pm 0.44 \mathrm{~b}$ & $2.07 \pm 0.42 \mathrm{~cd}$ & $1.37 \pm 0.31$ de & $0.65 \pm 0.08 \mathrm{~d}$ & $9.07 \pm 0.87 \mathrm{ab}$ & $0.77 \pm 0.09 \mathrm{e}$ & $1.25 \pm 0.21 \mathrm{~d}$ & $5.75 \pm 0.42 \mathrm{bc}$ & $1.25 \pm 0.13 \mathrm{c}$ \\
\hline Grass clippings & $17.85 \pm 0.55 \mathrm{ab}$ & $13.00 \pm 1.23 \mathrm{bc}$ & $\begin{array}{l}11.12 \pm 1.11 \\
\text { bcd }\end{array}$ & $7.65 \pm 0.86 \mathrm{ab}$ & $\begin{array}{l}11.12 \pm 0.98 \\
\mathrm{abc}\end{array}$ & $5.52 \pm 0.49 c$ & $3.50 \pm 0.57 \mathrm{ab}$ & $9.00 \pm 0.91 \mathrm{ab}$ & $4.10 \pm 0.19 c$ & $5.15 \pm 0.48 c$ & $6.87 \pm 0.88 \mathrm{bc}$ & $6.12 \pm 0.44 \mathrm{~b}$ \\
\hline $\begin{array}{l}\text { Living mulch } \\
\text { (lentil crop) }\end{array}$ & $19.32 \pm 1.23 \mathrm{ab}$ & $15.37 \pm 1.55 \mathrm{ab}$ & $\begin{array}{c}13.02 \pm 1.13 \\
\mathrm{abc}\end{array}$ & $12.75 \pm 1.07 \mathrm{ab}$ & $11.75 \pm 1.06 \mathrm{a}$ & $8.70 \pm 0.87 \mathrm{~b}$ & $5.20 \pm 0.87 \mathrm{a}$ & $12.07 \pm 0.83 \mathrm{ab}$ & $4.52 \pm 0.41 \mathrm{bc}$ & $6.50 \pm 0.79 b$ & $12.75 \pm 1.00 \mathrm{a}$ & $8.25 \pm 0.58 \mathrm{a}$ \\
\hline $\begin{array}{l}\text { Black plastic } \\
\text { mulch }\end{array}$ & $18.27 \pm 1.78 \mathrm{ab}$ & $6.37 \pm 0.89 \mathrm{e}$ & $7.50 \pm 0.76 \mathrm{~cd}$ & $5.47 \pm 0.37 \mathrm{ab}$ & $5.00 \pm 0.39 \mathrm{bcd}$ & $1.00 \pm 0.21 \mathrm{e}$ & $0.55 \pm 0.06 \mathrm{~d}$ & $6.57 \pm 0.55 \mathrm{ab}$ & $0.32 \pm 0.02 \mathrm{e}$ & $1.25 \pm 0.25 \mathrm{~d}$ & $4.00 \pm 0.52 \mathrm{c}$ & $0.50 \pm 0.04 \mathrm{c}$ \\
\hline \multirow[t]{2}{*}{$\begin{array}{l}\text { Herbicide spray } \\
\text { LSD at } 0.05 P\end{array}$} & $\begin{array}{c}22.42 \pm 1.99 \mathrm{a} \\
11.21\end{array}$ & $\begin{array}{c}8.12 \pm 0.99 \mathrm{de} \\
3.98\end{array}$ & $\begin{array}{c}5.75 \pm 0.44 \mathrm{~d} \\
6.37\end{array}$ & $\begin{array}{c}6.05 \pm 0.49 \mathrm{ab} \\
9.31\end{array}$ & $\begin{array}{c}1.00 \pm 0.19 \mathrm{~d} \\
9.71\end{array}$ & $\begin{array}{c}0.75 \pm 0.10 \mathrm{e} \\
1.81\end{array}$ & $\begin{array}{c}0.60 \pm 0.03 \mathrm{~d} \\
1.87\end{array}$ & $\begin{array}{c}3.12 \pm 0.19 \mathrm{~b} \\
10.87\end{array}$ & $\begin{array}{c}1.20 \pm \mathrm{e} \\
1.49\end{array}$ & $\begin{array}{c}1.75 \pm 0.31 \mathrm{~d} \\
1.34\end{array}$ & $\begin{array}{c}4.00 \pm 0.39 \mathrm{c} \\
4.94\end{array}$ & $\begin{array}{c}0.25 \pm 0.01 \mathrm{c} \\
1.80\end{array}$ \\
\hline & \multicolumn{3}{|c|}{ Euphorbia helioscopia } & \multicolumn{3}{|c|}{ Fumaria indica } & \multicolumn{3}{|c|}{ Phalaris minor } & \multicolumn{3}{|c|}{ Sonchus oleraceus } \\
\hline Control & $16.75 \pm 0.88 \mathrm{a}$ & $16.75 \pm 1.55 \mathrm{a}$ & $16.12 \pm 1.57 \mathrm{a}$ & $16.52 \pm 1.51 \mathrm{a}$ & $16.30 \pm 1.23 \mathrm{ab}$ & $15.67 \pm 1.66 \mathrm{a}$ & $18.12 \pm 1.47 \mathrm{a}$ & $17.70 \pm 1.26 \mathrm{a}$ & $17.75 \pm 1.25 \mathrm{a}$ & $12.15 \pm 1.58 \mathrm{a}$ & $8.12 \pm 1.01 \mathrm{a}$ & $9.72 \pm 1.36 \mathrm{a}$ \\
\hline $\begin{array}{l}\text { Maize stalk } \\
\text { mulch }\end{array}$ & $15.35 \pm 1.23 \mathrm{a}$ & $16.15 \pm 1.81 \mathrm{a}$ & $9.00 \pm 1.11 \mathrm{c}$ & $12.25 \pm 1.71 \mathrm{ab}$ & $10.0 \pm 1.05 \mathrm{bcd}$ & $7.62 \pm 0.82 b$ & $17.70 \pm 1.89 a$ & $\begin{array}{c}13.27 \pm 1.01 \\
\mathrm{abc}\end{array}$ & $11.12 \pm 1.61 \mathrm{~b}$ & $8.40 \pm 1.71 \mathrm{a}$ & $3.25 \pm 0.82 \mathrm{bc}$ & $2.37 \pm 0.41 c$ \\
\hline $\begin{array}{l}\text { Dry leaves of } \\
\text { mulberry }\end{array}$ & $16.07 \pm 1.54 \mathrm{a}$ & $15.30 \pm 0.87 \mathrm{a}$ & $15.75 \pm 1.33 \mathrm{ab}$ & $15.50 \pm 1.28 \mathrm{ab}$ & $17.62 \pm 1.58 \mathrm{a}$ & $15.20 \pm 1.98 \mathrm{a}$ & $12.97 \pm 1.25 \mathrm{a}$ & $15.02 \pm 1.19 \mathrm{ab}$ & $16.50 \pm 1.88 \mathrm{a}$ & $9.97 \pm 1.23 \mathrm{a}$ & $7.975 \pm 1.16 \mathrm{a}$ & $8.55 \pm 1.11 \mathrm{a}$ \\
\hline $\begin{array}{l}\text { Sugarcane } \\
\text { bagasse }\end{array}$ & $15.00 \pm 0.89 a$ & $10.25 \pm 1.06 \mathrm{~b}$ & $7.50 \pm 0.69 c$ & $8.50 \pm 0.88 \mathrm{bc}$ & $\begin{array}{c}13.57 \pm 1.39 \\
\mathrm{abc}\end{array}$ & $4.00 \pm 0.78 \mathrm{c}$ & $13.07 \pm 1.21 \mathrm{a}$ & $\begin{array}{l}10.37 \pm 0.98 \\
\text { bcd }\end{array}$ & $1.00 \pm 0.87 \mathrm{c}$ & $8.64 \pm 1.01 \mathrm{a}$ & $2.22 \pm 0.87 \mathrm{bc}$ & $0.50 \pm 0.11 \mathrm{c}$ \\
\hline $\begin{array}{l}\text { Living mulch } \\
\text { (lentil crop) }\end{array}$ & $15.67 \pm 1.21 \mathrm{a}$ & $6.00 \pm 0.55 \mathrm{~cd}$ & $\begin{array}{c}13.47 \pm 1.36 \\
\text { abc }\end{array}$ & $14.02 \pm 1.34 \mathrm{ab}$ & $15.95 \pm 1.56 \mathrm{ab}$ & $14.85 \pm 1.56 \mathrm{a}$ & $14.72 \pm 1.44 \mathrm{a}$ & $16.95 \pm 1.61 \mathrm{a}$ & $15.32 \pm 1.41 \mathrm{a}$ & $11.97 \pm 1.25 \mathrm{a}$ & $7.350 \pm 1.45 \mathrm{a}$ & $7.70 \pm 0.83 \mathrm{a}$ \\
\hline $\begin{array}{l}\text { Black plastic } \\
\text { mulch }\end{array}$ & $14.87 \pm 1.47 \mathrm{a}$ & $5.00 \pm 0.85 \mathrm{~cd}$ & $10.00 \pm 0.88 \mathrm{bc}$ & $10.77 \pm 1.02 \mathrm{ab}$ & $6.25 \pm 0.54 \mathrm{~d}$ & $1.50 \pm 0.25 c$ & $15.70 \pm 1.55 \mathrm{a}$ & $6.25 \pm 0.65 \mathrm{~d}$ & $6.25 \pm b 0.89 c$ & $8.55 \pm 1.02 \mathrm{a}$ & $2.30 \pm 0.67 \mathrm{bc}$ & $0.75 \pm 0.13 c$ \\
\hline $\begin{array}{l}\text { Herbicide spray } \\
\text { LSD at } 0.05 P\end{array}$ & $\begin{array}{c}12.97 \pm 1.29 \mathrm{a} \\
\mathrm{NS}\end{array}$ & $\begin{array}{c}3.75 \pm 0.71 \mathrm{~d} \\
3.95\end{array}$ & $\begin{array}{c}7.50 \pm 0.65 \mathrm{c} \\
6.04\end{array}$ & $\begin{array}{c}1.77 \pm 0.56 \mathrm{c} \\
7.51\end{array}$ & $\begin{array}{c}8.75 \pm 0.87 \mathrm{~cd} \\
6.72\end{array}$ & $\begin{array}{c}0.75 \pm 0.26 \mathrm{c} \\
3.03\end{array}$ & $\begin{array}{c}17.27 \pm 1.21 \mathrm{a} \\
\mathrm{NS}\end{array}$ & $\begin{array}{c}7.75 \pm 0.69 \mathrm{~cd} \\
6.04\end{array}$ & $\begin{array}{c}2.50 \pm 0.47 \mathrm{c} \\
4.15\end{array}$ & $\begin{array}{c}7.05 \pm 1.23 \mathrm{a} \\
\mathrm{NS}\end{array}$ & $\begin{array}{c}2.07 \pm 0.59 \mathrm{c} \\
2.39\end{array}$ & $\begin{array}{c}0.75 \pm 0.19 c \\
2.16\end{array}$ \\
\hline
\end{tabular}

The values represent the averages ( \pm SE) of three independent replicates followed by different letters within columns are significantly different at $p \leq 0.05$, according to Tukey's HSD test. 


\subsection{Mulching Materials Effect on Fresh Biomass}

Highest reduction in fresh biomass of all the weed species was observed under Herbicide spray at 25, 50 and 75 DAS respectively (Table 3). The other mulching treatments found effective in reducing fresh biomass were black plastic mulch followed by sugarcane bagasse, grass clipping, maize stalk mulch and living mulch respectively. Overall black plastic mulch and sugarcane bagasse were found as best mulching treatments to reduce the fresh biomass of all the investigated weed species at 25, 50 and 75 DAS (Table 3).

\subsection{Mulching Materials Effect on Dry Biomass}

Highest decrease in dry biomass of all the weed species was recorded under herbicide application (Table 4). Black plastic mulch and grass clipping followed by sugarcane bagasse were found as best treatments to reduce the dry biomass of all the tested weeds at 25,50 and 75 DAS. The effect of black plastic mulch and sugarcane bagasse in reducing dry biomass of Avena fatua and Carthamus oxyacantha was similar and statistically alike to herbicide treatment. Similarly, grass clippings and maize stalk mulch produced statistically similar effects in dry biomass reduction of Carthamus oxyacantha (Table 4).

\subsection{Mulching Materials Effect on Plant Height, Number of Leaves, Leaf Area, 1000-Grain Weight and Grain Yield of Wheat}

The effect of mulching treatments on wheat plant height was significant (Table 5). Maximum plant height of wheat was obtained where herbicide was sprayed followed by black plastic mulch. Minimum plant height was recorded where lentil was intercropped with wheat, however, it was statistically similar to dry leaves of mulberry and control where no mulch was applied. The numbers of leaves in wheat crops at 25, 50 and 75 DAS were significantly affected by mulching treatments. Wheat produced more number of leaves where herbicide was sprayed and it was statistically similar to black plastic mulch followed by sugarcane bagasse and maize stalk mulch. Similarly more leaf area of wheat was recorded where herbicide was sprayed and it was statistically similar to black plastic mulch followed by sugarcane bagasse. Minimum leaf area of wheat plants was noted where lentil was intercropped with wheat, however, it was statistically similar to dry leaves of mulberry and control (Table 5). The effects of mulching treatments on 1000 grain weight and grain yield of wheat were significant (Table 5, Figure 3). Maximum 1000 grain weight was obtained where sugarcane bagasse was applied followed by grass clipping mulch and dry leaves of mulberry. Minimum 1000 grain weight of wheat was recorded under control. Maximum wheat yield $\left(5.87 \mathrm{t} \mathrm{ha}^{-1}\right)$ was recorded where plastic mulch was used followed by herbicide spray $\left(5.81 \mathrm{tha}^{-1}\right)$ and dry leaves of mulberry $\left(5.72 \mathrm{t} \mathrm{ha}^{-1}\right)$. 


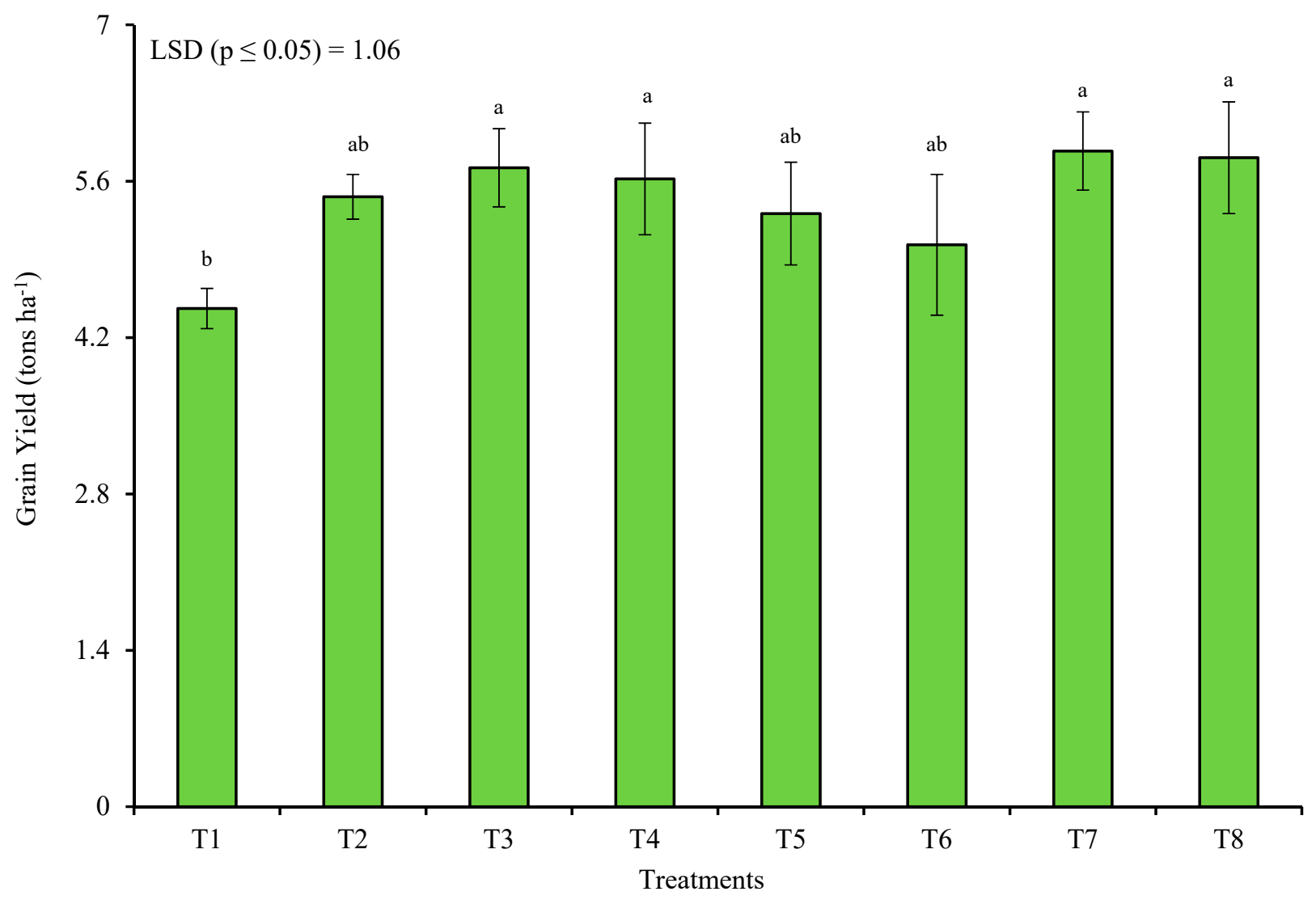

Figure 3. Effect of various mulch materials on grain yield of wheat. Data are the means of three replicates with standard deviations shown by vertical bars. T1 = Control, T2 = Maize stalk mulch, T3 = Dry leaves of mulberry, T4 = Sugarcane bagasse, T5 = Grass clippings, T6 = Living mulch (lentil crop), T7 = Black plastic mulch, T8 = herbicide (Buctril Super @ $1.235 \mathrm{~L} \mathrm{ha}^{-1}$ ). All different letters are significant at $p \leq 0.05$.

\subsection{Economic Analysis of Treatments}

The economic analysis indicated highest BCR (2.55) and net earnings (678 USD ha ${ }^{-1}$ ) where grass clippings were used as mulch followed by dry leaves of mulberry, sugarcane bagasse, control, mulch of black plastic and herbicide spray to combat weeds in wheat (Table 6). 
Table 3. Effect of mulch materials on fresh biomass ( $\mathrm{g}$ ) of different weed species of wheat.

\begin{tabular}{|c|c|c|c|c|c|c|c|c|c|c|c|c|}
\hline \multirow{2}{*}{ Treatments } & \multicolumn{3}{|c|}{ Avena fatua } & \multicolumn{3}{|c|}{ Carthamus oxyacantha } & \multicolumn{3}{|c|}{ Chenopodium album } & \multicolumn{3}{|c|}{ Convolvulus arvensis } \\
\hline & 25 DAS & 50 DAS & 75 DAS & 25 DAS & 50 DAS & 75 DAS & 25 DAS & 50 DAS & 75 DAS & 25 DAS & 50 DAS & 75 DAS \\
\hline Control & $7.05 \pm 1.09 \mathrm{a}$ & $14.55 \pm 1.07 \mathrm{a}$ & $24.92 \pm 2.17 \mathrm{a}$ & $4.90 \pm 0.63 \mathrm{a}$ & $12.05 \pm 0.86 \mathrm{a}$ & $17.25 \pm 1.21 \mathrm{a}$ & $5.80 \pm 0.77 \mathrm{a}$ & $14.87 \pm 1.23 \mathrm{a}$ & $16.20 \pm 1.56 \mathrm{a}$ & $7.92 \pm 0.79 \mathrm{a}$ & $8.07 \pm 1.11 \mathrm{a}$ & $16.25 \pm 1.59 \mathrm{a}$ \\
\hline \multirow{5}{*}{$\begin{array}{l}\text { Maize stalk mulch } \\
\text { Dry leaves of } \\
\text { mulberry } \\
\text { Sugarcane bagasse } \\
\text { Grass clippings } \\
\text { Living mulch } \\
\text { (lentil crop) } \\
\text { Black plastic } \\
\text { mulch } \\
\text { Herbicide spray } \\
\text { LSD at } 0.05 P\end{array}$} & $2.85 \pm 0.77 \mathrm{~cd}$ & $7.40 \pm 0.71 \mathrm{c}$ & $12.95 \pm 1.01 \mathrm{c}$ & $1.40 \pm 0.61 \mathrm{c}$ & $3.62 \pm 0.45 \mathrm{de}$ & $8.50 \pm 0.92 \mathrm{~cd}$ & $0.50 \pm 0.09 \mathrm{~d}$ & $5.15 \pm 0.59 c$ & $7.95 \pm 0.81 \mathrm{abcd}$ & $3.92 \pm 0.51 \mathrm{~cd}$ & $3.75 \pm 0.68 c$ & $8.95 \pm 1.23 \mathrm{ab}$ \\
\hline & $4.95 \pm 0.93 \mathrm{~b}$ & $12.47 \pm 1.00 \mathrm{ab}$ & $21.12 \pm 1.57 \mathrm{ab}$ & $2.52 \pm 0.77 \mathrm{~b}$ & $9.50 \pm 1.05 b$ & $10.00 \pm 1.06 \mathrm{bc}$ & $1.27 \pm 0.19 \mathrm{c}$ & $12.35 \pm 1.05 \mathrm{a}$ & $15.20 \pm 0.99 \mathrm{ab}$ & $5.10 \pm 0.46 \mathrm{~b}$ & $6.85 \pm 0.87 \mathrm{ab}$ & $13.82 \pm 0.95 \mathrm{ab}$ \\
\hline & $\begin{array}{l}1.95 \pm 0.44 \mathrm{~d} \\
4.05 \pm 0.29 \mathrm{bc}\end{array}$ & $\begin{array}{l}4.47 \pm 0.55 \mathrm{~d} \\
7.35 \pm 0.76 \mathrm{c}\end{array}$ & $\begin{array}{c}6.75 \pm 0.67 \mathrm{~d} \\
9.57 \pm 0.93 \mathrm{~cd}\end{array}$ & $\begin{array}{l}0.75 \pm 0.17 \mathrm{~cd} \\
0.97 \pm 0.49 \mathrm{~cd}\end{array}$ & $\begin{array}{l}0.57 \pm 0.41 \mathrm{e} \\
4.17 \pm 0.66 \mathrm{~d}\end{array}$ & $\begin{array}{l}4.75 \pm 0.56 \mathrm{~cd} \\
8.75 \pm 0.77 \mathrm{~cd}\end{array}$ & $\begin{array}{l}0.12 \pm 0.02 \mathrm{~d} \\
1.20 \pm 0.28 \mathrm{c}\end{array}$ & $\begin{array}{c}2.37 \pm 0.69 \mathrm{bc} \\
4.87 \pm 0.82 \mathrm{c}\end{array}$ & $\begin{array}{c}6.25 \pm 0.69 \mathrm{bcd} \\
11.00 \pm 0.86 \mathrm{abc}\end{array}$ & $\begin{array}{l}1.62 \pm 0.41 \mathrm{e} \\
3.07 \pm 0.48 \mathrm{~d}\end{array}$ & $\begin{array}{l}1.45 \pm 0.45 \mathrm{~d} \\
5.35 \pm 0.69 \mathrm{~b}\end{array}$ & $\begin{array}{l}4.25 \pm 0.82 \mathrm{~b} \\
9.50 \pm 1.03 \mathrm{ab}\end{array}$ \\
\hline & $5.17 \pm 0.61 \mathrm{ab}$ & $13.25 \pm 1.29 \mathrm{a}$ & $22.62 \pm 1.73 \mathrm{a}$ & $2.80 \pm 0.51 \mathrm{~b}$ & $8.17 \pm 0.89 \mathrm{~b}$ & $14.70 \pm 1.23 \mathrm{ab}$ & $2.65 \pm 0.54 \mathrm{~b}$ & $11.32 \pm 1.34 \mathrm{ab}$ & $14.70 \pm 1.02 \mathrm{ab}$ & $4.35 \pm 0.59 \mathrm{bc}$ & $6.07 \pm 0.88 \mathrm{a}$ & $13.82 \pm 1.56 \mathrm{a}$ \\
\hline & $1.47 \pm 0.28 \mathrm{~d}$ & $2.22 \pm 0.45 \mathrm{e}$ & $3.37 \pm 0.36 \mathrm{e}$ & $0.47 \pm 0.09 \mathrm{~d}$ & $0.62 \pm 0.41 \mathrm{e}$ & $5.00 \pm 0.72 \mathrm{~cd}$ & $0.10 \pm 0.01 \mathrm{~d}$ & $0.85 \pm 0.56 \mathrm{~d}$ & $5.00 \pm 0.56 \mathrm{~cd}$ & $1.60 \pm 0.66 \mathrm{e}$ & $1.00 \pm 0.16 \mathrm{~d}$ & $5.00 \pm 0.78 \mathrm{~b}$ \\
\hline & \multicolumn{3}{|c|}{ Euphorbia helioscopia } & \multicolumn{3}{|c|}{ Fumaria indica } & \multicolumn{3}{|c|}{ Phalaris minor } & \multicolumn{3}{|c|}{ Sonchus oleraceus } \\
\hline $\begin{array}{l}\text { Control } \\
\text { Maize stalk mulch }\end{array}$ & $\begin{array}{l}7.52 \pm 0.87 \mathrm{a} \\
4.75+0.75 \mathrm{~b}\end{array}$ & $\begin{array}{l}9.15 \pm 1.25 \mathrm{a} \\
3.42 \pm 0.46 \mathrm{~d}\end{array}$ & $23.07 \pm 1.81 \mathrm{a}$ & $5.57 \pm 0.49 \mathrm{a}$ & $\begin{array}{c}11.0 \pm 0.88 \mathrm{a} \\
4.7 \pm 0.51 \mathrm{~b}\end{array}$ & $\begin{array}{l}19.5 \pm 1.49 \mathrm{a} \\
5.42 \pm 0.88 \mathrm{~d}\end{array}$ & $\begin{array}{l}8.45 \pm 1.13 \mathrm{a} \\
4.40+0.55 \mathrm{bc}\end{array}$ & $14.40 \pm 0.96 \mathrm{a}$ & & $6.70 \pm 0.88 \mathrm{a}$ & $4.97 \pm 0.96 \mathrm{a}$ & $15.27 \pm 1.54 \mathrm{a}$ \\
\hline $\begin{array}{l}\text { Maize stalk mulch } \\
\text { Dry leaves of }\end{array}$ & & & $21.00 \pm 1.23 \mathrm{a}$ & $3.42 \pm 0.49 b$ & $\begin{array}{l}4.7 \pm 0.51 b \\
9.6 \pm 0.89 a\end{array}$ & $\begin{array}{l}5.42 \pm 0.88 \mathrm{~d} \\
15.10 \pm 1.11 \mathrm{~b}\end{array}$ & $\begin{array}{l}4.40 \pm 0.55 \mathrm{bc} \\
7.45 \pm 1.00 \mathrm{a}\end{array}$ & $\begin{array}{l}8.52 \pm 0.85 \mathrm{~b} \\
1272 \pm 0.90 \mathrm{a}\end{array}$ & & $\begin{array}{l}3.20 \pm 0.43 \mathrm{abc} \\
470 \pm 0.81 \mathrm{ab}\end{array}$ & $\begin{array}{l}1.15 \pm 0.73 \mathrm{~d} \\
3.47 \pm 0.91 \mathrm{~b}\end{array}$ & $\begin{array}{l}3.80 \pm 0.04 \mathrm{bc} \\
13.05+1.01 \mathrm{a}\end{array}$ \\
\hline $\begin{array}{l}\text { mulberry } \\
\text { Sugarcane bagasse }\end{array}$ & $1.32 \pm 0.41 \mathrm{c}$ & $1.35 \pm 0.47 \mathrm{e}$ & $3.07 \pm 0.22 \mathrm{~b}$ & $1.17 \pm 0.56 \mathrm{bc}$ & $2.12 \pm 0.77 \mathrm{c}$ & $3.00 \pm 0.52 \mathrm{e}$ & $2.82 \pm 0.49 \mathrm{~cd}$ & $3.80 \pm 0.65 \mathrm{c}$ & $2.80 \pm 0.55 \mathrm{~d}$ & $1.22 \pm 0.23 \mathrm{bc}$ & $0.40 \pm 0.17 \mathrm{~d}$ & $1.42 \pm 0.56 \mathrm{bc}$ \\
\hline Grass clippings & $3.32 \pm 0.22 \mathrm{bc}$ & $4.72 \pm 0.54 \mathrm{~cd}$ & $5.22 \pm 0.79 \mathrm{~b}$ & $1.55 \pm 0.59 c$ & $5.30 \pm 0.21 \mathrm{~b}$ & $7.22 \pm 0.87 c$ & $6.07 \pm 0.78 \mathrm{ab}$ & $6.12 \pm 0.41 c$ & $8.10 \pm 0.99 b$ & $1.65 \pm 0.22 \mathrm{bc}$ & $2.57 \pm 0.76 \mathrm{bc}$ & $4.57 \pm 0.87 \mathrm{~b}$ \\
\hline $\begin{array}{l}\text { Living mulch } \\
\text { (lentil crop) }\end{array}$ & $5.10 \pm 0.69 \mathrm{ab}$ & $6.05 \pm 0.79 \mathrm{bc}$ & $21.00 \pm 1.49 \mathrm{a}$ & $3.00 \pm 0.76 \mathrm{~b}$ & $9.77 \pm 0.96 \mathrm{a}$ & $16.02 \pm 1.01 \mathrm{~b}$ & $8.35 \pm 1.17 \mathrm{a}$ & $12.35 \pm 1.03 \mathrm{a}$ & $19.50 \pm 1.87 \mathrm{a}$ & $3.97 \pm 0.57 \mathrm{ab}$ & $3.00 \pm 0.86 \mathrm{~b}$ & $12.05 \pm 0.91 \mathrm{a}$ \\
\hline $\begin{array}{l}\text { Black plastic } \\
\text { mulch }\end{array}$ & $1.27 \pm 0.17 \mathrm{c}$ & $1.20 \pm 0.36 \mathrm{e}$ & $3.35 \pm 0.52 \mathrm{~b}$ & $0.62 \pm 0.09 \mathrm{~d}$ & $2.80 \pm 0.21 \mathrm{bc}$ & $2.90 \pm 0.57 \mathrm{e}$ & $1.90 \pm 0.11 \mathrm{~d}$ & $1.20 \pm 0.23 \mathrm{~d}$ & $0.25 \pm 0.07 \mathrm{~d}$ & $0.95 \pm 0.71 \mathrm{c}$ & $0.45 \pm 0.09 \mathrm{~d}$ & $1.32 \pm 0.21 \mathrm{bc}$ \\
\hline
\end{tabular}

The values represent the averages ( \pm SE) of three independent replicates followed by different letters within columns are significantly different at $p \leq 0.05$, according to Tukey's HSD test. 
Table 4. Effect of various mulch materials on dry biomass ( $\mathrm{g}$ ) of different weed species of wheat.

\begin{tabular}{|c|c|c|c|c|c|c|c|c|c|c|c|c|}
\hline \multirow{2}{*}{ Treatments } & \multicolumn{3}{|c|}{ Avena fatua } & \multicolumn{3}{|c|}{ Carthamus oxyacantha } & \multicolumn{3}{|c|}{ Chenopodium album } & \multicolumn{3}{|c|}{ Convolvulus arvensis } \\
\hline & 25 DAS & 50 DAS & 75 DAS & 25 DAS & 50 DAS & 75 DAS & 25 DAS & 50 DAS & 75 DAS & 25 DAS & 50 DAS & 75 DAS \\
\hline Control & $2.27 \pm 0.58 \mathrm{a}$ & $4.47 \pm 0.89 \mathrm{a}$ & $8.47 \pm 1.96 \mathrm{a}$ & $2.50 \pm 0.87 a$ & $4.95 \pm 1.25 \mathrm{a}$ & $6.25 \pm 1.87 \mathrm{a}$ & $9.02 \pm 1.73 \mathrm{a}$ & $5.52 \pm 1.41 \mathrm{a}$ & $20.57 \pm 2.22 \mathrm{a}$ & $2.60 \pm 0.41 \mathrm{a}$ & $3.90 \pm 0.88 \mathrm{a}$ & $6.20 \pm 1.25 a$ \\
\hline Maize stalk mulch & $1.50 \pm 0.39 \mathrm{abc}$ & $2.02 \pm 0.61 \mathrm{de}$ & $2.72 \pm 0.75 \mathrm{de}$ & $1.05 \pm 0.45 \mathrm{~cd}$ & $1.50 \pm 0.58 \mathrm{~d}$ & $2.05 \pm 0.85 c$ & $2.57 \pm 0.56 \mathrm{~d}$ & $1.10 \pm 0.59 \mathrm{de}$ & $8.00 \pm 0.91 \mathrm{~b}$ & $1.62 \pm 0.24 \mathrm{bc}$ & $1.13 \pm 0.54 \mathrm{c}$ & $2.17 \pm 0.52 \mathrm{~d}$ \\
\hline Dry leaves of mulberry & $1.82 \pm 0.41 \mathrm{ab}$ & $3.42 \pm 0.96 \mathrm{ab}$ & $5.57 \pm 1.17 \mathrm{c}$ & $1.80 \pm 0.69 \mathrm{~b}$ & $3.35 \pm 0.89 \mathrm{bc}$ & $4.37 \pm 0.99 \mathrm{~b}$ & $6.25 \pm 1.19 \mathrm{~b}$ & $2.72 \pm 0.87 \mathrm{bc}$ & $18.15 \pm 1.47 \mathrm{a}$ & $2.30 \pm 0.59 \mathrm{ab}$ & $2.00 \pm 0.59 \mathrm{~b}$ & $3.57 \pm 0.98 \mathrm{c}$ \\
\hline Grass clippings & $0.67 \pm 0.17 \mathrm{~cd}$ & $2.15 \pm 0.78 \mathrm{~cd}$ & $3.57 \pm 0.91 \mathrm{~d}$ & $0.15 \pm 0.11 \mathrm{e}$ & $2.55 \pm 0.85 \mathrm{~cd}$ & $3.77 \pm 1.11 \mathrm{~b}$ & $5.12 \pm 0.83 \mathrm{bc}$ & $2.07 \pm 0.49 \mathrm{~cd}$ & $9.47 \pm 1.01 \mathrm{~b}$ & $1.55 \pm 0.63 c$ & $2.25 \pm 0.87 \mathrm{~b}$ & $3.00 \pm 0.87 \mathrm{~cd}$ \\
\hline $\begin{array}{l}\text { Living mulch (lentil } \\
\text { crop) }\end{array}$ & $1.95 \pm 0.39 \mathrm{a}$ & $3.17 \pm 0.75 \mathrm{bc}$ & $6.95 \pm 1.65 b$ & $1.55 \pm 0.68 \mathrm{bc}$ & $4.07 \pm 1.07 \mathrm{ab}$ & $6.35 \pm 1.39 \mathrm{a}$ & $5.00 \pm 1.18 \mathrm{c}$ & $3.75 \pm 0.77 b$ & $19.72 \pm 2.03 \mathrm{a}$ & $2.00 \pm 0.55 \mathrm{a}$ & $3.35 \pm 0.95 \mathrm{a}$ & $5.00 \pm 1.09 \mathrm{~b}$ \\
\hline Black plastic mulch & $0.77 \pm 0.14 \mathrm{~cd}$ & $0.80 \pm 0.27 \mathrm{f}$ & $0.40 \pm 0.29 \mathrm{f}$ & $0.15 \pm 0.09 \mathrm{e}$ & $0.12 \pm 0.05 \mathrm{e}$ & $0.55 \pm 0.21 \mathrm{~d}$ & $1.00 \pm 0.41 \mathrm{e}$ & $0.42 \pm 0.08 \mathrm{e}$ & $0.95 \pm 0.29 c$ & $0.22 \pm 0.04 \mathrm{~d}$ & $0.55 \pm 0.12 c$ & $0.45 \pm 0.19 \mathrm{e}$ \\
\hline Herbicide spray & $0.32 \pm 0.07 \mathrm{~d}$ & $0.70 \pm 0.21 \mathrm{f}$ & $0.32 \pm 0.21 \mathrm{f}$ & $0.17 \pm 0.08 \mathrm{e}$ & $0.13 \pm 0.04 \mathrm{e}$ & $0.35 \pm 0.19 \mathrm{~d}$ & $1.12 \pm 0.49 \mathrm{e}$ & $0.12 \pm 0.02 \mathrm{e}$ & $0.00 \pm 0.00 c$ & $0.27 \pm 0.06 \mathrm{~d}$ & $0.37 \pm 0.09 c$ & $0.25 \pm 0.08 \mathrm{e}$ \\
\hline \multirow[t]{2}{*}{ LSD at $0.05 P$} & 1.08 & 1.10 & 1.25 & 0.70 & 1.25 & 1.30 & 1.24 & 1.13 & 4.27 & 0.77 & 0.86 & 1.00 \\
\hline & \multicolumn{3}{|c|}{ Euphorbia helioscopia } & \multicolumn{3}{|c|}{ Fumaria indica } & \multicolumn{3}{|c|}{ Phalaris minor } & \multicolumn{3}{|c|}{ Sonchus oleraceus } \\
\hline Control & $2.22 \pm 0.81 \mathrm{a}$ & $4.00 \pm 0.89 \mathrm{a}$ & $6.12 \pm 1.07 \mathrm{a}$ & $3.22 \pm 0.95 \mathrm{a}$ & $5.17 \pm 1.08 \mathrm{a}$ & $7.72 \pm 1.25 \mathrm{a}$ & $3.25 \pm 1.09 \mathrm{a}$ & $4.57 \pm 1.05 \mathrm{a}$ & $7.67 \pm 1.54 \mathrm{a}$ & $2.20 \pm 0.75 \mathrm{a}$ & $7.52 \pm 1.23 \mathrm{a}$ & $6.25 \pm 1.10 \mathrm{a}$ \\
\hline Maize stalk mulch & $1.42 \pm 0.65 \mathrm{ab}$ & $1.27 \pm 0.77 \mathrm{bc}$ & $2.02 \pm 0.88 \mathrm{c}$ & $0.80 \pm 0.15 \mathrm{c}$ & $1.57 \pm 0.45 \mathrm{~d}$ & $1.80 \pm 0.54 \mathrm{~d}$ & $1.42 \pm 0.87 \mathrm{bc}$ & $2.12 \pm 0.87 \mathrm{~b}$ & $2.45 \pm 0.81 \mathrm{~d}$ & $0.97 \pm 0.29 \mathrm{bc}$ & $1.25 \pm 0.27 \mathrm{~d}$ & $1.25 \pm 0.57 \mathrm{c}$ \\
\hline Dry leaves of mulberry & $2.05 \pm 0.61 \mathrm{a}$ & $2.17 \pm 0.82 \mathrm{~b}$ & $4.25 \pm 0.99 \mathrm{~b}$ & $1.82 \pm 0.68 \mathrm{bc}$ & $3.72 \pm 0.88 \mathrm{~b}$ & $5.30 \pm 0.93 \mathrm{~b}$ & $2.05 \pm 0.99 \mathrm{~b}$ & $3.77 \pm 0.99 \mathrm{a}$ & $5.17 \pm 1.01 \mathrm{bc}$ & $1.87 \pm 0.70 \mathrm{ab}$ & $5.67 \pm 1.21 \mathrm{~b}$ & $3.50 \pm 0.86 \mathrm{~b}$ \\
\hline Sugarcane bagasse & $0.22 \pm 0.23 \mathrm{~cd}$ & $0.75 \pm 0.40 c$ & $0.82 \pm 0.31 \mathrm{~d}$ & $0.60 \pm 0.21 \mathrm{~cd}$ & $0.47 \pm 0.19 \mathrm{e}$ & $0.00 \pm 0.00 \mathrm{e}$ & $0.52 \pm 0.19 \mathrm{~cd}$ & $0.95 \pm 0.35 c$ & $0.00 \pm 0.0 \mathrm{e}$ & $0.32 \pm 0.04 \mathrm{c}$ & $1.00 \pm 0.38 \mathrm{~d}$ & $0.57 \pm 0.17 c$ \\
\hline Grass clippings & $1.02 \pm 0.77 \mathrm{bc}$ & $2.10 \pm 0.65 \mathrm{~b}$ & $2.40 \pm 0.78 \mathrm{c}$ & $0.20 \pm 0.09 \mathrm{de}$ & $2.70 \pm 0.69 \mathrm{c}$ & $3.97 \pm 1.00 \mathrm{c}$ & $1.55 \pm 0.55 \mathrm{bc}$ & $2.25 \pm 0.81 \mathrm{~b}$ & $3.92 \pm 0.85 \mathrm{~cd}$ & $0.27 \pm 0.05 \mathrm{c}$ & $3.55 \pm 0.57 \mathrm{c}$ & $2.75 \pm 0.69 \mathrm{~b}$ \\
\hline Living mulch (lentil & $1.60 \pm 0.78 \mathrm{a}$ & $3.40 \pm 0.99 \mathrm{a}$ & $5.20 \pm 0.83 \mathrm{ab}$ & $2.07 \pm 0.45 \mathrm{~b}$ & $4.17 \pm 0.86 \mathrm{~b}$ & $7.25 \pm 1.34 \mathrm{a}$ & $1.25 \pm 0.61 \mathrm{c}$ & $4.17 \pm 1.09 \mathrm{a}$ & $6.72 \pm 1.22 \mathrm{ab}$ & $1.65 \pm 0.45 \mathrm{ab}$ & $7.00 \pm 0.98 \mathrm{ab}$ & $5.50 \pm 0.94 \mathrm{a}$ \\
\hline Black plastic mulch & $0.17 \pm 0.11 \mathrm{~d}$ & $0.67 \pm 0.21 \mathrm{c}$ & $0.57 \pm 0.19 \mathrm{~d}$ & $0.10 \pm 0.01 \mathrm{e}$ & $0.55 \pm 0.09 \mathrm{e}$ & $0.00 \pm 0.00 \mathrm{e}$ & $0.40 \pm 0.11 \mathrm{~d}$ & $0.57 \pm 0.19 c$ & $0.15 \pm 0.03 \mathrm{e}$ & $0.12 \pm 0.02 c$ & $1.00 \pm 0.28 \mathrm{~d}$ & $0.42 \pm 0.14 \mathrm{c}$ \\
\hline Herbicide spray & $0.12 \pm 0.09 \mathrm{~d}$ & $0.52 \pm 0.19 c$ & $0.37 \pm 0.09 \mathrm{~d}$ & $0.10 \pm 0.01 \mathrm{e}$ & $0.32 \pm 0.06 \mathrm{e}$ & $0.00 \pm 0.00 \mathrm{e}$ & $0.20 \pm 0.05 \mathrm{~d}$ & $0.55 \pm 0.13 \mathrm{c}$ & $0.35 \pm 0.09 \mathrm{e}$ & $0.12 \pm 0.01 \mathrm{c}$ & $0.75 \pm 0.19 \mathrm{~d}$ & $0.40 \pm 0.10 \mathrm{c}$ \\
\hline LSD at $0.05 P$ & 0.84 & 1.14 & 1.03 & 0.40 & 0.57 & 0.99 & 0.75 & 1.10 & 1.88 & 0.91 & 1.74 & 1.43 \\
\hline
\end{tabular}

The values represent the averages ( \pm SE) of three independent replicates followed by different letters within columns are significantly different at $p \leq 0.05$, according to Tukey's HSD test. 
Table 5. Effect of various mulch materials on growth and yield of wheat.

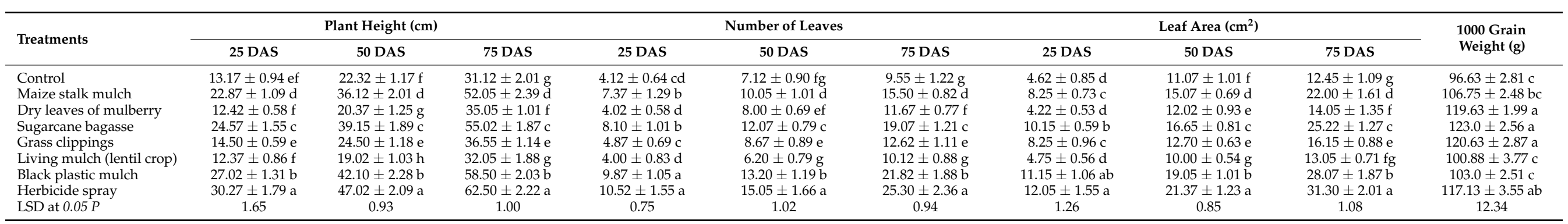

The values represent the averages $( \pm$ SE) of three independent replicates followed by different letters within columns are significantly different at $p \leq 0.05$, according to Tukey's HSD test.

Table 6. Economic analysis of mulching treatments for weed suppression in wheat during 2013-2014 and 2014-2015.

\begin{tabular}{|c|c|c|c|c|c|c|c|c|c|}
\hline Parameters & T1 & T2 & T3 & T4 & T5 & T6 & T7 & T8 & Remarks \\
\hline Grain yield & 3.10 & 3.25 & 4.10 & 4.06 & 4.08 & 3.36 & 3.40 & 4.09 & $\mathrm{tha}^{-1}$ \\
\hline Adjusted yield & 2.79 & 2.92 & 3.69 & 3.65 & 3.67 & 3.02 & 3.06 & 3.69 & Less than $10 \%$ from actual yield \\
\hline Value & 698.55 & 732.49 & 924.06 & 915.05 & 924.71 & 757.29 & 766.3 & 921.81 & USD 10.02 per $40 \mathrm{~kg}$ \\
\hline Value of wheat straw & 147.87 & 155.03 & 195.57 & 193.66 & 194.62 & 160.27 & 162.18 & 195.09 & USD 1.91 per $40 \mathrm{~kg}$ \\
\hline Gross benefits & 846.55 & 887.52 & 1119.63 & 1108.71 & 1114.18 & 917.56 & 928.48 & 1116.91 & \\
\hline Cost of maize stalk mulch & & 30.53 & & & & & & & USD 4.77 per $\mathrm{kg}$ \\
\hline Cost of dry leaves of mulberry & & & 33.39 & & & & & & Aeration pump \& container rent @ USD \\
\hline Cost of sugarcane bagasse & & & & 41.02 & & & & & $\begin{array}{l}0.48 \text { per day } \\
\text { USD ha }^{-1}\end{array}$ \\
\hline Cost of grass clippings & & & & & 20.99 & & & & USD ha $^{-1}$ \\
\hline Cost of living mulch & & & & & & 118.77 & & & $\mathrm{USD} \mathrm{ha}^{-1}$ \\
\hline Cost of black plastic mulch & & & & & & & 42.93 & & $\mathrm{USD} \mathrm{ha}^{-1}$ \\
\hline Cost of herbicide & & & & & & & & 161.13 & USD ha $^{-1}$ \\
\hline Permanent cost & 414.51 & 414.51 & 414.51 & 414.51 & 414.51 & 414.51 & 414.51 & 414.51 & USD ha $^{-1}$ \\
\hline Total expenditure & 414.51 & 445.04 & 447.9 & 455.54 & 435.5 & 533.29 & 457.44 & 575.64 & USD ha $^{-1}$ \\
\hline Net benefits & 432.04 & 442.47 & 671.73 & 6531.8 & 678.68 & 384.27 & 471.04 & 541.26 & USD ha $^{-1}$ \\
\hline Benefit cost ratio (BCR) & 2.04 & 1.99 & 2.49 & 2.43 & 2.55 & 1.72 & 2.02 & 1.94 & \\
\hline
\end{tabular}

$\mathrm{T} 1=$ Control,
$\left.1.2351 \mathrm{ha}^{-1}\right)$. 


\section{Discussion}

The effect of various mulch materials on relative weed density, weed density, biomass of weed species in wheat was significant in the current research study. Notable reduction in relative weed density, weed density, and fresh and dry biomass of weed species was noticed where herbicide and black plastic mulch were used followed by sugarcane bagasse in comparison to control (no mulch was used). Maximum relative weed density, weed density, and weed biomass was observed where lentil was intercropped with wheat and dry leaves of mulberry applications which may be due to the phytotoxic effects of herbicide. Application of herbicides provides quick outcomes by blocking energy production units of weed plants. Herbicides also affect cellular respiration of weed plants. Reduction in relative weed density, weed density, fresh and dry biomass of wheat weeds using mulch of black plastic are in line with Ashrafuzzaman et al. [22] who reported that black plastic mulch could result in $100 \%$ control of all weeds in maize. Kareem et al. [23] reported that mulch of black plastic progressively reduced weeds density in okra plants. Mulches reduce evaporation and erosion of soil as well as cause reduction in weed growth and increase crop yield in different crops [24,25]. Mulching helps in reducing soil evaporation, conserving moisture, controlling soil temperature, reducing weed growth, and improving microbial activities [26]. Grass clippings, dry leaves of mulberry and sugarcane bagasse decreased relative weed density, weed density, and biomass of weeds in wheat crops. Reduction in weeds density and their fresh and dry biomass by these mulching treatments might be due to the presence of allelochemicals which have phytotoxic effects on weeds. Earlier, Sampietro et al. [27] reported that sugarcane straw had trans-ferulic, cis-ferulic, vanillic and syringic acids. The allelochemicals of phenolic nature increase leakage of root cell constituents, inhibited dehydrogenase activity and reduced chlorophyll content in lettuce. In an another research study, biomass production in soyabean plants biomass reduced because of low chlorophyll content when they were treated with phenolic acids (vanillic acids, ferulic, $p$-coumaric) [28]. The increase in relative weed density, biomass of weed species were recorded in the current study, where lentil (living mulch) was intercropped with wheat. High weed density and relative weed density might be due to competition for essential resources (nutrients, moisture and light). Majority of weeds belong to $\mathrm{C}_{4}$ category of plants which are efficient to $\mathrm{CO}_{2}$ and absorb nutrients. Moreover, weeds have deep root systems and uptake moisture and nutrients more deeply than other $C_{3}$ plants such as wheat and lentil. Weeds have high photosynthetic rates due to high carboxylation rate. Higher leaf area in wheat due to herbicide, plastic mulch and sugarcane bagasse applications might have led to higher dry matter accumulation in the crop on account of higher radiation interception over a prolonged period of time. Better resource acquisition and utilization might have favored wheat growth, higher tillers, spikes, more dry matter accumulation and higher 1000-grain weight by wheat plants. Our results conform with those of Chhokar et al., [29] and Santos [30] who reported that herbicides offer a sizeable increase in crop productivity corresponding to their weed control spectrum. Sugarcane bagasse had allelochemicals which are toxic to weeds, reduce weeds growth, their density, and hence increased 1000 grain yield in wheat. Other possible reasons behind increasing the wheat grain yield may be due to increase in soil water availability by sugarcane bagasse applications. Sugarcane bagasse on its decomposition produces organic acids, which mobilize the insoluble phosphorus from soil to soil solution in the available form. In an earlier research, sugarcane bagasse not only improved the physical condition of the soil but also enhanced macro-spore for a better root growth, and ultimately improved the cane yield [31]. These results also confirmed the findings of Lamont [32] who found that sugarcane bagasse is good option for better crop yield. Kader et al. [33] and Wang et al. $[34,35]$ found that mulching is beneficial for yield enhancement with reduced input resources. Nwosisi et al. [36] revealed similar results with organic mulching in sweet potato cultivars. 


\section{Conclusions}

Based on reduction in weed density, relative weed density, fresh biomass, dry biomass of different weed species of wheat and corresponding positive influence on plant height, number of leaves, leaf area and 1000-grain weight of wheat it was concluded that grass clippings and sugarcane bagasse each used at $4 \mathrm{t} \mathrm{ha}^{-1}$ as a mulch and easily available provided effective weed control in wheat. Therefore, this can be used for decreasing weed dynamics in wheat under rain-fed conditions with reduced cost of production in Haripur, Pakistan.

Author Contributions: S.U.K. (Sami Ullah Khan) conceived the idea. T.M. conducted the experiment. S.L. and S.U.K. (Saftain Ullah Khan) collected the literature review. S.U.K. (Sami Ullah Khan) and A.Q. provided technical expertise to strengthen the basic idea. S.U.K. (Saftain Ullah Khan) X.W., S.F. and A.Q. helped in statistical analysis. S.F. and A.Q. proofread and provided intellectual guidance. All authors read the first draft, helped in revision and approved the article. All authors have read and agreed to the published version of the manuscript.

Funding: The publication of the present work is supported by the National Key Research and Development Program of China (grant no. 2017YFC0504704) and the National Natural Science Foundation of China $(51669034,41761068,51809224)$.

Institutional Review Board Statement: Not applicable.

Informed Consent Statement: Not applicable.

Data Availability Statement: Not applicable.

Conflicts of Interest: The authors declare that there are no conflicts of interest.

\section{References}

1. Zafar, S.; Ashraf, M.Y.; Niaz, M.; Kausar, A.; Hussain, J. Evaluation of wheat genotypes for salinity tolerance using physiological indices as screening tool. Pak. J. Bot. 2015, 47, 397-405.

2. Razzaq, A.; Cheema, Z.A.; Jabran, K.; Hussain, M.; Farooq, M.; Zafar, M. Reduced herbicide doses used together with allelopathic sorghum and sunflower water extracts for weed control in wheat. J. Plant Prot. Res. 2012, 52, 281-285. [CrossRef]

3. Sadeghi, A.M.; Isensee, A.R.; Shirmohammadi, A. Influence of soil texture and tillage on herbicide transport. Chemosphere 2000, 41, 1327-1332. [CrossRef]

4. Ma, Y. Allelopathic studies of common wheat (Triticum aestivum L.). Weed Biol. Manag. 2005, 5, 93-104. [CrossRef]

5. Jabran, K. Manipulation of Allelopathic Crops for Weed Control; Springer International Publishing: Cham, Swizterland, 2017.

6. Mehmood, T.; Khan, S.; Qayyum, A.; Gurmani, A.R.; Ahmed, W.; Liaquat, M.; Farid, A. Evaluation of Organic and Inorganic Mulching as an Integrated Weed Management Strategy in Maize Under Rainfed Conditions. Planta Daninha 2018, 36, e018184892. [CrossRef]

7. Huerta 2018, A.; Chiffelle, I.; Puga, K.; Azúa, F.; Araya, J.E. Toxicity and repellence of aqueous and ethanolic extracts from Schinus molle on elm leaf beetle Xanthogaleruca luteola. Crop Prot. 2010, 29, 1118-1123. [CrossRef]

8. Jabran, K.; Farooq, M.; Hussain, M.; Rehman, H.; Ali, M.A. Wild oat (Avena fatua L.) and canary grass (Phalaris minor Ritz.) management through allelopathy. J. Plant Prot. Res. 2010, 50, 32-33. [CrossRef]

9. Farooq, M.; Bajwa, A.A.; Cheema, S.A.; Cheema, Z.A. Application of allelopathy in crop production. Int. J. Agric. Biol. 2013, 15, 1367-1378.

10. Arif, M.; Cheema, Z.A.; Hassan, A. Organic weed management in wheat through allelopathy. Int. J. Agric. Biol. 2015, 17, 127-134.

11. Tabaglio, V.; Gavazzi, C.; Schulz, M.; Marocco, A. Alternative weed control using the allelopathic effect of natural benzoxazinoids from rye mulch. Agron. Sustain. Dev. 2008, 28, 397-401. [CrossRef]

12. Mirsky, S.B.; Ryan, M.R.; Teasdale, J.R.; Curran, W.S.; Reberg-Horton, C.S.; Spargo, J.T.; Wells, M.S.; Keene, C.L.; Moyer, J.W. Overcoming weed management challenges in cover crop-based organic rotational no-till soybean production in the eastern United States. Weed Technol. 2013, 27, 193-203. [CrossRef]

13. Khan, I.A.; Rehman, O.U.; Khan, S.A.; Samady, H.A.; Alzahrani, Y. Effect of Different Herbicides, Plant Extracts and Mulches on Yield and Yield Components of Maize. Planta Daninha 2020, 38, e02019. [CrossRef]

14. Cheema, Z.A.; Sadiq, H.M.I.; Khaliq, A. Efficacy of sorgaab (sorghum water extract) as a natural weed inhibitor in wheat. Int. J. Agric. Biol. 2000, 2, 144-146.

15. Ryan, J.; Rashid, A. Application of soil and plant analysis for applied research and development in West Asia-North Africa: An international center's perspective. Commun. Soil Sci. Plant Anal. 2006, 37, 2185-2198. [CrossRef]

16. Soltanpour, P.N.; Schwab, A.P. A new soil test for simultaneous extraction of macro and micronutrients in alkaline soils. Commun. Soil Sci. Plant Anal. 1977, 8, 195-207. [CrossRef] 
17. Bremner, J.M. Nitrogen Total. In Methods of Soil Analysis Part 3: Chemical Methods; Sparks, D.L., Ed.; SSSA Book Series 5; Soil Science Society of America: Madison, WI, USA, 1996; pp. 1085-1122.

18. Ball, D.F. Loss-on-ignition as an estimate of organic matter and organic carbon in non-calcareous soils. J. Soil Sci. 1964, 15, 84-92. [CrossRef]

19. Lindsay, W.L.; Norvell, W.A. Development of a DTPA soil test for zinc, iron, manganese, and copper. Soil Sci. Soc. Am. J. 1978, 42, 421-428. [CrossRef]

20. Lancashire, P.D.; Bleiholder, H.; Langeluddecke, P.; Stauss, R.; Van den Boom, T.; Weber, E.; Witzen-Berger, A. A uniform decimal code for growth stages of crops and weeds. Ann. Appl. Biol. 1991, 119, 561-601. [CrossRef]

21. CIMMYT Economics Program, International Maize, \& Wheat Improvement Center from Agronomic Data to Farmer Recommendations: An Economics Training Manual (No. 27); CIMMYT: Mexico City, Mexico, 1988.

22. Ashrafuzzaman, M.; Halim, M.A.; Ismail, M.R.; Shahidullah, S.M.; Hossain, M. Effect of plastic mulch on growth and yield of chilli (Capsicum annuum L.). Braz. Arch. Biol. Technol. 2011, 54, 321-330. [CrossRef]

23. Kareem, K.T.; Alamu, O.O.; Egberongbe, R.K.; Arogundade, O. Effect of different mulch materials on the incidence and severity of okra mosaic virus (OMV) in okra plants. J. Appl. Hortic. 2012, 14, 1-14. [CrossRef]

24. Li, S.X.; Wang, Z.H.; Li, S.Q.; Gao, Y.J.; Tian, X.H. Effect of plastic sheet mulch, wheat straw mulch, and maize growth on water loss by evaporation in dryland areas of China. Agric. Water Manag. 2013, 116, 39-49. [CrossRef]

25. Qin, S.; Zhang, J.; Dai, H.; Wang, D.; Li, D. Effect of ridge-furrow and plastic-mulching planting patterns on yield formation and water movement of potato in a semi-arid area. Agric. Water Manag. 2014, 131, 87-94. [CrossRef]

26. Cheng, F.; Cheng, Z. Research progress on the use of plant allelopathy in agriculture and the physiological and ecological mechanisms of allelopathy. Front. Plant Sci. 2015, 6, 1020. [CrossRef] [PubMed]

27. Sampietro, D.A.; Vattuone, M.A.; Isla, M.I. Plant growth inhibitors isolated from sugarcane (Saccharum officinarum) straw. J. Plant Physiol. 2006, 163, 837-846. [CrossRef] [PubMed]

28. Singh, Y.P.; Singh, G.; Sharma, D.K. Biomass and bio-energy production of ten multipurpose tree species planted in sodic soils of indo-gangetic plains. J. For. Res. 2010, 21, 19-24. [CrossRef]

29. Chhokar, R.S.; Singh, S.; Sharma, R.K. Herbicides for control of isoproturon-resistant Little seed Canary grass (Phalaris minor) in wheat. Crop Prot. 2008, 27, 719-726. [CrossRef]

30. Santos, B.M. Drip-applied metam potassium and herbicides as methyl bromide alternatives for Cyperus control in tomato. Crop Prot. 2009, 28, 68-71. [CrossRef]

31. Kameyama, K.; Miyamoto, T.; Shinogi, Y. Increases in available water content of soils by applying bagasse-charcoals. In Proceedings of the 19th World Congress of Soil Science, Soil Solutions for a Changing World, Brisbane, Australia, 1-6 August 2010; pp. 105-108.

32. Dotaniya, M.L.; Datta, S.C.; Biswas, D.R.; Dotaniya, C.K.; Meena, B.L.; Rajendiran, S.; Regar, K.L.; Lata, M. Use of sugarcane industrial by-products for improving sugarcane productivity and soil health. Int. J. Recycl. Org. Waste Agric. 2016, 5, 185-194. [CrossRef]

33. Kader, M.A.; Singha, A.; Begum, M.A.; Jewel, A.; Khan, F.H.; Khan, N.I. Mulching as water-saving technique in dryland agriculture. Bull. Natl. Res. Cent. 2019, 43,1-6. [CrossRef]

34. Wang, X.; Wang, G.; Go, T.; Fan, J.; Zhang, F. Effects of plastic mulch and nitrogen fertilizer on the soil microbial community, enzymatic activity and yield performance in a dryland maize cropping system. Eur. J. Soil Sci. 2021, 72, 400-412. [CrossRef]

35. Wang, X.; Fan, J.; Xing, Y.; Xu, G.; Wang, H.; Deng, J.; Wang, Y.; Zhang, F.; Li, P.; Li, Z. The effects of mulch and nitrogen fertilizer on the soil environment of crop plants. Adv. Agron. 2019, 153, 121-173.

36. Nwosisi, S.; Nandwani, D.; Hui, D. Mulch treatment effect on weed biomass and yields of organic sweet potato cultivars. Agronomy 2019, 9, 190. [CrossRef] 